\title{
Robust Geometric Formation Control of Multiple Autonomous Underwater Vehicles with Time Delays
}

\author{
Huizhen Yang, Chuanfeng Wang, and Fumin Zhang
}

\begin{abstract}
This paper develops a robust controller for autonomous underwater vehicles with bounded time delays, so that the AUVs form and keep a desired formation shape and track a desired trajectory. We use a six-degree-of-freedom dynamic model for each AUV to describe its motions in the three-dimensional space. We design an orientation controller based on feedback linearization, so that the orientation of each AUV converges to its desired value. We derive formation dynamics of AUVs and decouple the dynamics into a formation shape and a formation center, using the Jacobi transform. We treat couplings in the formation dynamics as perturbations and design a robust formation-keeping controller to tolerate both the perturbations and the time delays. We demonstrate the effectiveness of our controller in simulations.
\end{abstract}

\section{INTRODUCTION}

Formation control of autonomous underwater vehicles (AUVs)( [1]-[9]) is challenging due to the complex dynamics and communication constraints among AUVs. An AUV has complex dynamics which cannot be easily simplified in formation control design. In addition, underwater communication and positioning, which rely heavily on acoustic systems, are plagued with limited communication bandwidth, intermittent failures, latency and multi-path effects. Therefore, time delays in communication among AUVs should be considered for formation controller design. References [6], [8], [10], [11] considered the complex dynamics of an AUV and developed various control strategies for multi-AUV systems, without considering communication time delays. To integrate communication and control, [12] proposed a discrete-time Kuramoto model over one-to-all and all-toall logical graphs and analyzed its stability for a collection of identical planar unit-speed vehicles described by a twodimensional Frenet-Serret motion model. Using Lyapunov theory and a switching communication topology, [13] developed a coordinated path-following controller to deal with communication failures between AUVs. Reference [14] proposed a path-following control strategy to coordinate a group of surface vessels, which moves only in the horizontal plane. Graph theory was used in [15] and resulted in a cooperative

H. Yang is with the School of Marine, Northwestern Polytechnical University, Xi' an, China. rainsun_ly@nwpu . edu.cn. Her research is supported by NWPU 2011JC0242.

C. Wang is with the George W. Woodruff School of Mechanical Engineering, Georgia Institute of Technology, Atlanta, GA 30332, USA. cwang329@gatech.edu.

F. Zhang is with the School of Electrical and Computer Engineering, Georgia Institute of Technology, Atlanta, GA 30332, USA fuminegatech.edu. His research is supported by ONR grants N00014-08-1-1007, N00014-09-1-1074, and N00014-10-10712 (YIP), and NSF grants ECCS-0841195 (CAREER), CNS-0931576, and ECCS1056253 control law which was proved robust to small communication delays. In our previous paper [16], we studied formation dynamics for a group of AUVs in a horizontal plane. Using Jacobi coordinates, we expressed the formation dynamics as a deformable body and designed $H_{\infty}$ full state feedback controllers, assuming the time delays negligible.

The main contribution of this paper is that we extended the Jacobi transform approach from the two-dimensional horizontal plane to the three-dimensional space and used it to analyze the formation dynamics for a group of six-degreeof-freedom (6DOF) AUVs with time delays. According to the formation dynamics we derived, we designed a robust controller to achieve both path following and formation keeping. Our method splits the orientation subsystem of each AUV from the formation system, so both orientation and formation subsystems can be controlled separately. Our method also decouples the formation shape and the formation center, therefore, they can be controlled separately, too. The robust formation controller we designed stabilizes the formation system with a bounded time delay.

The rest of this paper is organized as follows. Section II reviews a 6DOF dynamic model of a single AUV and decouples it into a translational and a rotational part. An orientation controller is designed based on feedback linearization according to the rotational dynamics. In section III, applying the translational dynamics of each AUV, we derive the formation dynamics of AUVs in the three-dimensional space through Jacobi transform, propose a feedback decoupling for the formation dynamics, and design a robust formation controller to stabilize the formation system with perturbations and time delays. We give simulation results in Section IV and the conclusion in Section V.

\section{DYNAMICS AND ORIENTATION CONTROL OF A SINGLE AUV}

\section{A. Dynamic Model of A Single AUV}

We assume that every AUV in the formation system is fully actuated, homogeneous, and neutrally buoyant, so the center of buoyancy coincides with the center of gravity. We set the origin of the body-fixed frame at the center of buoyancy and assume that the hydrodynamic forces and moments are linear. $\eta_{1}=[x, y, z]^{T}$ denotes the AUV position in the earth-fixed frame. $\eta_{2}=[\phi, \theta, \psi]^{T}$ denotes the Eulerangle vector for roll, pith, and yaw in earth-fixed frame. $v_{1}=[u, v, w]^{T}$ denotes the body-fixed linear velocity for surge, sway, and heave. $v_{2}=[p, q, r]^{T}$ denotes the body-fixed angular velocity for roll, pith, and yaw. Then the dynamics 
of each AUV can be expressed as follows [17], [18]:

$$
\begin{aligned}
& \dot{\eta}_{1}=J_{1}\left(\eta_{2}\right) v_{1}, \\
& \dot{\eta}_{2}=J_{2}\left(\eta_{2}\right) v_{2}, \\
& M \dot{v}+C(v) v+D(v) v=\tau,
\end{aligned}
$$

where $v=\left[v_{1}^{T}, v_{2}^{T}\right]^{T}$, and $\tau=\left[\begin{array}{cc}\tau_{1}^{T} & \tau_{2}^{T}\end{array}\right]^{T}$ is the control inputs vector where $\tau_{1}^{T}$ is the controlling force vector and $\tau_{2}^{T}$ the controlling moment vector. In this model, $\left[\begin{array}{cc}J_{1}\left(\eta_{2}\right) & 0 \\ 0 & J_{2}\left(\eta_{2}\right)\end{array}\right]$ is the coordinate-transform (Jacobian) matrix from the body-fixed frame to the earth-fixed frame. The matrices $M$ and $D$ in this model are inertia and damping matrices, defined as

$$
\begin{aligned}
& M=\operatorname{diag}\left\{m-\mathscr{X}_{\dot{u}}, m-\mathscr{Y}_{\dot{v}}, m-\mathscr{Z}_{\dot{w}}, I_{x}, I_{y}, I_{z}\right\}, \\
& D(v)=-\operatorname{diag}\left\{\mathscr{X}_{u}, \mathscr{Y}_{v}, \mathscr{Z}_{w}, \mathscr{K}_{p}, \mathscr{M}_{q}, \mathscr{N}_{r}\right\} .
\end{aligned}
$$

where, $\mathscr{X}_{\dot{u}}, \mathscr{Y}_{\dot{v}}, \mathscr{Z}_{w}, I_{x}, I_{y}, I_{z}, \mathscr{X}_{u}, \mathscr{Y}_{v}, \mathscr{Z}_{w}, \mathscr{K}_{p}, \mathscr{M}_{q}$, and $\mathscr{N}_{r}$ are model parameters.

The matrix $C(v)$ represents the Coriolis and centripetal term, which contains the rigid-body Coriolis and centripetal term $C_{R B}(v)$ and the hydrodynamic added Coriolis and centripetal term $C_{A}(v)$, i.e.,

$$
C(v)=C_{R B}(v)+C_{A}(v),
$$

where

$C_{R B}(v)=\left[\begin{array}{ccc|ccc}0 & 0 & 0 & 0 & m w & -m v \\ 0 & 0 & 0 & -m w & 0 & m u \\ 0 & 0 & 0 & m v & -m u & 0 \\ \hline 0 & m w & -m v & 0 & I_{z} r & -I_{y} q \\ -m w & 0 & m u & -I_{z} r & 0 & I_{x} p \\ m v & -m u & 0 & I_{y} q & I_{x} p & 0\end{array}\right]_{(7)}$

$$
C_{A}(v)=
$$

$$
\left[\begin{array}{ccc|ccc}
0 & 0 & 0 & 0 & \mathscr{Z}_{\dot{w}} w & -\mathscr{Y}_{\dot{i}} v \\
0 & 0 & 0 & -\mathscr{Z}_{\dot{w} w} & 0 & \mathscr{X}_{\dot{u}} u \\
0 & 0 & 0 & \mathscr{Y}_{\dot{v} v} & -\mathscr{X}_{\dot{u}} u & 0 \\
\hline 0 & \mathscr{Z}_{\dot{w}} w & -\mathscr{Y}_{\dot{j}} v & 0 & -\mathscr{N}_{\dot{r}} r & \mathscr{M}_{\dot{q}} q \\
-\mathscr{Z}_{\dot{w}} w & 0 & \mathscr{X}_{\dot{u}} u & \mathscr{N}_{\dot{r}} r & 0 & -\mathscr{K}_{\dot{p}} p \\
\mathscr{Y}_{\dot{v}} v & -\mathscr{X}_{\dot{u}} u & 0 & -\mathscr{M}_{\dot{q}} q & \mathscr{K}_{\dot{p}} p & 0
\end{array}\right](8)
$$

Note that the $C_{R B}(v)$ and $C_{A}(v)$ satisfy the following property:

$$
\begin{aligned}
& C_{R B}^{\prime}(v) v=C_{R B}(v) v \\
& C_{A}^{\prime}(v) v=C_{A}(v) v .
\end{aligned}
$$

where,

$$
C_{R B}^{\prime}(v)=\left[\begin{array}{ccc|ccc}
0 & -m r & m q & 0 & 0 & 0 \\
m r & 0 & -m q & 0 & 0 & 0 \\
-m q & m p & 0 & 0 & 0 & 0 \\
\hline 0 & m w & -m v & 0 & I_{z} r & -I_{y} q \\
-m w & 0 & m u & -I_{z} r & 0 & I_{x} p \\
m v & -m u & 0 & I_{y} q & I_{x} p & 0
\end{array}\right]_{10}
$$

$$
\begin{aligned}
& C_{A}^{\prime}(v)= \\
& {\left[\begin{array}{ccc|ccc}
0 & \mathscr{Y}_{\dot{v}} r & -\mathscr{Z}_{\dot{w}} q & 0 & 0 & 0 \\
-\mathscr{X}_{\dot{u}} r & 0 & \mathscr{Z}_{\dot{w}} p & 0 & 0 & 0 \\
\mathscr{X}_{\dot{u}} q & -\mathscr{Y}_{\dot{v}} p & 0 & 0 & 0 & 0 \\
\hline 0 & \mathscr{Z}_{\dot{w}} w & -\mathscr{Y}_{\dot{v}} v & 0 & -\mathscr{N}_{\dot{r}} r & \mathscr{M}_{\dot{q}} q \\
-\mathscr{Z}_{\dot{w}} w & 0 & \mathscr{X}_{\dot{u}} u & \mathscr{N}_{\dot{r}} r & 0 & -\mathscr{K}_{\dot{p}} p \\
\mathscr{Y}_{\dot{v}} v & -\mathscr{X}_{\dot{u}} u & 0 & -\mathscr{M}_{\dot{q}} q & \mathscr{K}_{\dot{p}} p & 0
\end{array}\right] .}
\end{aligned}
$$

Now we can rewrite term $C(v) v+D(v) v$ in (3) as

$$
\begin{aligned}
& C(v) v+D(v) v=C_{R B}^{\prime}(v) v+C_{A}^{\prime}(v) v+D(v) v \\
& =\left[\begin{array}{c|c}
C_{11}\left(v_{2}\right) & 0 \\
\hline C_{21}\left(v_{1}\right) & C_{22}\left(v_{2}\right)
\end{array}\right] v \doteq C^{\prime}(v) v .
\end{aligned}
$$

where

$$
\begin{aligned}
C_{11}\left(v_{2}\right)= & {\left[\begin{array}{ccc}
-\mathscr{X}_{u} & -m r+\mathscr{Y}_{\dot{v}} r & m q-\mathscr{Z}_{\dot{w}} q \\
m r-\mathscr{X}_{\dot{u}} r & -\mathscr{Y}_{v} & -m p+\mathscr{Z}_{\dot{w}} p \\
-m q+\mathscr{X}_{\dot{u}} q & m p-\mathscr{Y}_{\dot{v}} p & -\mathscr{Z}_{w}
\end{array}\right], } \\
C_{21}\left(v_{1}\right)= & {\left[\begin{array}{ccc}
0 & m w+\mathscr{Z}_{\dot{w}} w & -m v+\mathscr{Y}_{\dot{v}} v \\
-m w-\mathscr{Z}_{\dot{w}} w & 0 & m u-\mathscr{X}_{\dot{u}} u \\
m v-\mathscr{Y}_{\dot{v}} v & -m u+\mathscr{X}_{\dot{u}} u & 0
\end{array}\right], } \\
C_{22}\left(v_{2}\right)= & {\left[\begin{array}{ccc}
-\mathscr{K}_{p} & I_{z} r-\mathscr{N}_{\dot{r}} r & -I_{y} q+\mathscr{M}_{\dot{q}} q \\
-I_{z} r+\mathscr{N}_{\dot{r}} r & -\mathscr{M}_{q} & I_{x} p-\mathscr{K}_{\dot{p}} p \\
I_{y} q-\mathscr{M}_{\dot{q}} q & -I_{x} p+\mathscr{K}_{\dot{p}} p & -\mathscr{N}_{r}
\end{array}\right] . }
\end{aligned}
$$

Therefore, (3) can be decomposed in the following two equations:

$$
\begin{aligned}
& M_{1} \dot{v}_{1}+C_{11}\left(v_{2}\right) v_{1}=\tau_{1}, \\
& M_{2} \dot{v}_{2}+C_{21}\left(v_{1}\right) v_{1}+C_{22}\left(v_{2}\right) v_{2}=\tau_{2},
\end{aligned}
$$

where

$$
\begin{aligned}
& M_{1}=\operatorname{diag}\left\{m-\mathscr{X}_{\dot{u}}, m-\mathscr{Y}_{\dot{v}}, m-\mathscr{Z}_{\dot{w}}\right\}, \\
& M_{2}=\operatorname{diag}\left\{I_{x}, I_{y}, I_{z}\right\} .
\end{aligned}
$$

Note that dynamics of an AUV can be fully described by (1), (2), (16) and (17). Eq.(1) and (16) describe the translational dynamics and (2) and (17) describe the orientation dynamics for a single AUV.

\section{B. Orientation Dynamics And Control}

Rewrite (17) as

$$
\dot{v}_{2}=-M_{2}^{-1} C_{22}\left(v_{2}\right) V_{2}+M_{2}^{-1} \tau_{2}-M_{2}^{-1} C_{21}\left(v_{1}\right) v_{1},
$$

and make the following invertible transformation for $\tau_{2}$

$$
\varsigma=M_{2}^{-1} \tau_{2}-M_{2}^{-1} C_{21}\left(v_{1}\right) v_{1},
$$

then we get

$$
\dot{v}_{2}=-M_{2}^{-1} C_{22}\left(v_{2}\right) v_{2}+\varsigma,
$$

where $C_{21}\left(v_{1}\right)$ and $C_{22}\left(v_{2}\right)$ are given in (14) and (15).

Define error vectors $E_{\eta_{2}}=\eta_{2}-\eta_{2 d}$ and $E_{v_{2}}=v_{2}-v_{2 d}$, where $\eta_{2 d}$ and $v_{2 d}$ are desired orientation angle and angular speed, respectively, and assume $\dot{\eta}_{2 d}=0$ and $\dot{v}_{2 d}=0$, which means that the desired orientation angle and angular speed 
are constant. By rewriting (2) and (21), we get the following state equation for $E_{\eta_{2}}$ and $E_{v_{2}}$ :

$$
\left\{\begin{array}{l}
\dot{E}_{\eta_{2}}=J_{2}\left(E_{\eta_{2}}+\eta_{2 d}\right)\left[E_{v_{2}}+v_{2 d}\right] \\
\dot{E}_{v_{2}}=-M_{2}^{-1} C_{22}\left(E_{v_{2}}+v_{2 d}\right)\left[E_{v_{2}}+v_{2 d}\right]+\varsigma
\end{array}\right.
$$

Now define the following diffeomorphism to achieve inputoutput linearization,

$$
\left\{\begin{array}{l}
z_{1} \doteq E_{\eta_{2}} \\
z_{2} \doteq \dot{z}_{1}=J_{2}\left(E_{\eta_{2}}+\eta_{2 d}\right)\left[E_{v_{2}}+v_{2 d}\right]
\end{array}\right.
$$

then the orientation dynamics become

$$
\left\{\begin{array}{l}
\dot{z}_{1}=z_{2} \\
\dot{z}_{2}=J_{2}\left(z_{1}+\eta_{2 d}\right)\left[-M_{2}^{-1} C_{22}\left(J_{2}^{-1}\left(z_{1}+\eta_{2 d}\right) z_{2}\right)\right. \\
\left.\left[J_{2}^{-1}\left(z_{1}+\eta_{2 d}\right) z_{2}\right]-\frac{d J_{2}^{-1}\left(z_{1}+\eta_{2 d}\right)}{d t} z_{2}+\varsigma\right] .
\end{array}\right.
$$

Choose the control law as

$$
\begin{aligned}
& \varsigma=M_{2}^{-1} C_{22}\left(J_{2}^{-1}\left(z_{1}+\eta_{2 d}\right) z_{2}\right)\left[J_{2}^{-1}\left(z_{1}+\eta_{2 d}\right) z_{2}\right] \\
& +\frac{d J_{2}^{-1}\left(z_{1}+\eta_{2 d}\right)}{d t} z_{2}+J_{2}^{-1}\left(z_{1}+\eta_{2 d}\right)\left[k_{1} z_{1}+k_{2} z_{2}\right]
\end{aligned}
$$

then the orientation subsystem becomes

$$
\left\{\begin{array}{l}
\dot{z}_{1}=z_{2} \\
\dot{z}_{2}=k_{1} z_{1}+k_{2} z_{2}
\end{array}\right.
$$

The state feedback gains $k_{1}$ and $k_{2}$ that make $\left[\begin{array}{cc}0 & 1 \\ k_{1} & k_{2}\end{array}\right]$ Hurwitz will stabilize the orientation system, therefore $E_{\eta_{2}}=$ $z_{1} \rightarrow 0$ as $t \rightarrow \infty$, which implies that the orientation converges to the desired value.

\section{AUV FORMATION DYNAMICS AND CONTROL}

\section{A. Translational Dynamics of A Single AUV}

From (1), we get $v_{1}=J_{1}^{-1}\left(\eta_{2}\right) \dot{\eta}_{1}$ and then

$$
\dot{v}_{1}=J_{1}^{-1}\left(\eta_{2}\right) \ddot{\eta}_{1}-J_{1}^{-1}\left(\eta_{2}\right) \dot{J}_{1}\left(\eta_{2}\right) J_{1}^{-1}\left(\eta_{2}\right) \dot{\eta}_{1}
$$

Substitute $v_{1}=J_{1}^{-1}\left(\eta_{2}\right) \dot{\eta}_{1}$ and (27) into (16), we obtain

$$
\ddot{\eta}_{1}=G\left(\eta_{2}, v_{2}\right) \dot{\eta}_{1}+H\left(\eta_{2}\right) \tau_{1}
$$

where

$$
G\left(\eta_{2}, v_{2}\right)=\dot{J}_{1}\left(\eta_{2}\right) J_{1}^{-1}\left(\eta_{2}\right)-J_{1}\left(\eta_{2}\right) M_{1}^{-1} C_{11}\left(v_{2}\right) J_{1}^{-1}\left(\eta_{2}\right)
$$

and

$$
H\left(\eta_{2}\right)=J_{1}\left(\eta_{2}\right) M_{1}^{-1}
$$

Now (28) describes the complete translational dynamics of each AUV. We use it to derive the formation dynamics of AUVs.

\section{B. Formation Dynamics of AUVs}

We consider a formation of $N$ AUVs moving in the threedimensional space. Let $\eta_{1}^{i}=\left[x_{i}, y_{i}, z_{i}\right]^{T}$ denote the position of the $i$-th AUV, where $i=1,2, \ldots, N$, then the Jacobi vectors are defined by a linear transform $\Omega$ that produces the following equation [16], [19]:

$$
\left[\rho_{1}^{T}, \rho_{2}^{T}, \ldots, \rho_{N-1}^{T}, q_{c}^{T}\right]^{T}=\Omega\left[\left(\eta_{1}^{1}\right)^{T},\left(\eta_{1}^{2}\right)^{T}, \ldots,\left(\eta_{1}^{N}\right)^{T}\right]^{T},
$$

where $\rho_{j}, j=1,2, \ldots, N-1$, are $N-1$ independent Jacobi vectors describing the geometric formation shape and $q_{c}$ is the formation center defined by $q_{c}=\frac{1}{N} \sum_{i=1}^{N} \eta_{1}^{i}$. The linear transform $\Omega$ is guaranteed to exist [19]. Our goal is to design a formation control to guarantee

$$
\rho_{j} \rightarrow \rho_{j d}, \dot{\rho}_{j} \rightarrow \dot{\rho}_{j d}, q_{c} \rightarrow q_{c d}, \dot{q}_{c} \rightarrow \dot{q}_{c d},
$$

where $\rho_{j d}$ is the desired value of the $j$-th Jacobi vector and $q_{c d}$ is the desired trajectory of the formation center.

Take second-order derivatives on both sides of (29), we get

$$
\left[\ddot{\rho}_{1}^{T}, \ddot{\rho}_{2}^{T}, \ldots, \ddot{\rho}_{N-1}^{T}, \ddot{q}_{c}^{T}\right]^{T}=\Omega\left[\left(\ddot{\eta}_{1}^{1}\right)^{T},\left(\ddot{\eta}_{1}^{2}\right)^{T}, \ldots,\left(\ddot{\eta}_{1}^{N}\right)^{T}\right]^{T} .
$$

Plug (28) into (31), and define state vector

$$
X=\left[\rho_{1}^{T}, \ldots, \rho_{N-1}^{T}, q_{c}^{T}\right]^{T},
$$

we obtain the formation dynamics as follows:

$$
\ddot{X}=A\left(\left[\eta_{2}^{i}\right],\left[v_{2}^{i}\right]\right) \dot{X}+\Gamma\left(\left[\eta_{2}^{i}\right]\right) U,
$$

where

$$
\begin{aligned}
& A\left(\left[\eta_{2}^{i}\right],\left[v_{2}^{i}\right]\right)=\Omega \mathbf{G} \Omega^{-1}, \\
& \mathbf{G}=\operatorname{diag}\left\{G_{1}\left(\eta_{2}^{1}, v_{2}^{1}\right), \ldots, G_{N}\left(\eta_{2}^{N}, v_{2}^{N}\right)\right\}, \\
& \Gamma\left(\left[\eta_{2}^{i}\right]\right)=\Omega \operatorname{diag}\left\{H\left(\eta_{2}^{1}\right), \ldots, H\left(\eta_{2}^{N}\right)\right\}, \\
& U=\left[\begin{array}{lll}
\left(\tau_{1}^{1}\right)^{T} & \cdots & \left(\tau_{1}^{N}\right)^{T}
\end{array}\right]^{T} .
\end{aligned}
$$

\section{Decoupling of the Formation Dynamics}

We have shown that the formation dynamics are described by (33), in which matrix $A$ is a nonlinear function of $p_{i}, q_{i}, r_{i}, \phi_{i}, \theta_{i}$, and $\psi_{i}$, for $i=1,2, \cdots, N$. We decompose $A$ into the following two parts:

$$
A\left(\left[\eta_{2}^{i}\right],\left[v_{2}^{i}\right]\right)=A_{\lambda}+A_{\Delta}\left(\left[\eta_{2}^{i}\right],\left[v_{2}^{i}\right]\right),
$$

where $A_{\lambda}=\lambda I_{3 N}$ is a constant diagonal matrix and $A_{\Delta}$ is viewed as a perturbation. We will find a $\lambda$ such that the $H_{\infty}$ norm of $A_{\Delta}$ is bounded and minimized.

Note that the perturbation term $A_{\Delta}$ is caused by asymmetries of an AUV. For an AUV with three planes of symmetry, $\mathscr{X}_{\dot{u}}=\mathscr{Y}_{\dot{v}}=\mathscr{Z}_{\dot{w}}$ and $\mathscr{X}_{u}=\mathscr{Y}_{v}=\mathscr{Z}_{w}$, which will make $\mathbf{G}$ a constant diagonal matrix, and then $A=A_{\lambda}=\frac{\mathscr{X}_{u}}{m-\mathscr{X}_{\dot{u}}} I_{3 N}$, therefore, $A_{\Delta}=0_{3 N}$, i.e., the perturbation term vanishes.

For a generic AUV, we select

$$
\lambda=\min \left(\frac{\mathscr{X}_{u}}{m-\mathscr{X}_{\dot{u}}}, \frac{\mathscr{Y}_{v}}{m-\mathscr{Y}_{\dot{v}}}, \frac{\mathscr{Z}_{w}}{m-\mathscr{Z}_{\dot{w}}}\right),
$$

then the $H_{\infty}$ norm of $A_{\Delta}$ is minimized. Define $\sigma^{2}=$ $\lambda_{\text {max }}\left(A_{\Delta}^{T} A_{\Delta}\right)$, then from (34) and (35) and all entries of $\mathbf{G}$, we can see that $\sigma$ is a function of $p_{i}, q_{i}, r_{i}$, and sine and 
cosine functions of $\theta_{i}, \phi_{i}$, and $\psi_{i}$. Because $p_{i}, q_{i}$, and $r_{i}$ are bounded as the vehicle can not steer infinitely fast, and sine and cosine functions are also bounded, we get $\sigma<\infty$.

\section{Stability Analysis of Time-delay Formation System}

Define $Z=\dot{X}$ and plug (38) into (33), we get

$$
\left\{\begin{array}{l}
\dot{X}(t)=Z(t) \\
\dot{Z}(t)=A_{\lambda} Z+A_{\Delta}\left(\left[\eta_{2}^{i}\right],\left[v_{2}^{i}\right]\right) Z+\Gamma\left(\left[\eta_{2}^{i}\right]\right) U .
\end{array}\right.
$$

Define error vectors $X_{e}=X-X_{d}$ and $Z_{e}=Z-Z_{d}$, where $X_{d}$ and $Z_{d}$ are desired values, and a perturbation term $W=A_{\Delta} Z_{e}$, we get

$$
\left\{\begin{array}{l}
\dot{X}_{e}(t)=Z_{e}(t) \\
\dot{Z}_{e}(t)=A_{\lambda} Z_{e}(t)+W(t)+\mu(t),
\end{array}\right.
$$

where

$$
\mu(t)=\Gamma\left(\left[\eta_{2}^{i}\right]\right) U(t)+A_{\lambda} Z_{d}+A_{\Delta}\left(\left[\eta_{2}^{i}\right],\left[v_{2}^{i}\right]\right) Z_{d}-\dot{Z}_{d}
$$

is the control we need to design, which will be a function of error vectors $X_{e}$ and $Z_{e}$. To compute $X_{e}$ and $Z_{e}$, position and velocity information of all AUVs are needed. As it takes time for the controller to get these information through communication links, the control effort is actually computed based on delayed information, i.e., $\mu(t)$ is a function of $X_{e}(t-h)$ and $Z_{e}(t-h)$. Here $h$ is a time delay satisfying $0 \leq h \leq h^{*}$ and $\dot{h}=0$, where $h^{*}$ is the finite upper bound of the time delay.

We rewrite (41) as

$$
\left\{\begin{array}{l}
\dot{\bar{X}}(t)=\bar{A} \bar{X}(t)+D W(t)+B_{h} \mu(t) \\
Z_{e}(t)=L \bar{X}(t)
\end{array}\right.
$$

where

$$
\begin{aligned}
& \bar{X}=\left[\begin{array}{ll}
X_{e}^{T} & Z_{e}^{T}
\end{array}\right]^{T}, \bar{A}=\left[\begin{array}{cc}
0 & I \\
0 & A_{\lambda}
\end{array}\right], \mu(t)=F \bar{X}(t-h), \\
& D=B_{h}=\left[\begin{array}{ll}
0 & I
\end{array}\right]^{T}, F=\left[\begin{array}{ll}
K_{2} & K_{1}
\end{array}\right], L=\left[\begin{array}{ll}
0 & I
\end{array}\right] .
\end{aligned}
$$

Theorem 1: Suppose the time delay $h$ satisfies $0 \leq h \leq h^{*}$ and $\dot{h}=0$. The full formation system (43) is delay-dependent stable and $\left\|T_{Z_{e} W}\right\| \leq \gamma$, where $\gamma>0$,

If there exists a positive symmetry matrix $Y=Y^{T}>0$ and scalars $\varepsilon_{1}>0, \varepsilon_{2}>0, \varepsilon_{3}>0$ satisfying $h^{*} \varepsilon_{3}-\gamma^{2}<0$ and the following linear matrix inequality(LMI):

$$
\left[\begin{array}{cccc}
\Phi & Y \bar{A}^{T} & Y F^{T} B_{h}^{T} & Y L^{T} \\
\bar{A} Y & -\frac{1}{h^{*} \varepsilon_{1}} I & 0 & 0 \\
B_{h} F Y & 0 & -\frac{1}{h^{*} \varepsilon_{2}} I & 0 \\
L Y & 0 & 0 & -I
\end{array}\right]<0
$$

where $\Phi=\left(\bar{A}+B_{h} F\right) Y+Y\left(\bar{A}+B_{h} F\right)^{T}+h^{*}\left(\varepsilon_{1}^{-1}+\varepsilon_{2}^{-1}+\right.$ $\left.\varepsilon_{3}^{-1}\right) B_{h} F F^{T} B_{h}^{T}+\frac{1}{\gamma^{2}-h^{*} \varepsilon_{3}} D D^{T}$.

Proof 1: Define $P=Y^{-1}$, then $P=P^{T}>0$ as $Y=Y^{T}>0$. Define the following Lyapunov-Krasovskii functional:

$$
\begin{aligned}
V_{1}[\bar{X}(t)] & =\bar{X}^{T}(t) P \bar{X}(t)+\int_{-h}^{0} \int_{t+\theta}^{t} \varepsilon_{1}\left[\bar{X}^{T}(s) \bar{A}^{T} \bar{A} \bar{X}(s)\right] d s d \theta \\
& +\int_{-h}^{0} \int_{t-h+\theta}^{t} \varepsilon_{2}\left[\bar{X}^{T}(s) F^{T} B_{h}^{T} B_{h} F \bar{X}(s)\right] d s d \theta \\
& +\int_{-h}^{0} \int_{t-h+\theta}^{t} \varepsilon_{3}\left[W^{T}(s) D^{T} D W(s)\right] d s d \theta
\end{aligned}
$$

Take derivative of $V_{1}[\bar{X}(t)]$ and use the following matrix-type inequality [20],

$$
\Sigma_{1}^{T} \Sigma_{2}+\Sigma_{2}^{T} \Sigma_{1} \leq \varepsilon \Sigma_{1}^{T} \Sigma_{1}+\varepsilon^{-1} \Sigma_{2}^{T} \Sigma_{2},
$$

where $\Sigma_{1}$ and $\Sigma_{2}$ are real constant matrices of compatible dimensions and $\varepsilon>0$ is a scalar, we get

$$
\begin{aligned}
& \dot{V}_{1}[\bar{X}(t)] \leq x^{T}(t)\left[P\left(\bar{A}+B_{h} F\right)+\left(\bar{A}+B_{h} F\right)^{T} P+h^{*} \varepsilon_{1} \bar{A}^{T} \bar{A}+\right. \\
& \left.h^{*} \varepsilon_{2} F^{T} B_{h}^{T} B_{h} F+h^{*}\left(\varepsilon_{1}^{-1}+\varepsilon_{2}^{-1}+\varepsilon_{3}^{-1}\right) P B_{h} F F^{T} B_{h}^{T} P\right] \bar{X}(t) \\
& +W^{T}(t) D^{T} P \bar{X}(t)+\bar{X}^{T}(t) P D W(t)+h^{*} r_{3} W^{T}(t) D^{T} D W(t)
\end{aligned}
$$

We define Hamiltonian as $H(\bar{X}, W, t)=\dot{V}_{1}[\bar{X}(t)]+$ $Z_{e}^{T}(t) Z_{e}(t)-\gamma^{2} W^{T}(t) D^{T} D W(t)$. Plug (46) into $H(\bar{X}, W, t)$, we get

$$
\begin{aligned}
H(\bar{X}, W, t) \leq & \bar{X}^{T}(t) \Phi_{2} \bar{X}(t)+W^{T}(t) D^{T} P W(t) \\
& +\bar{X}^{T}(t) P D W(t)+h^{*} \varepsilon_{3} W^{T}(t) D^{T} D W(t) \\
& -\gamma^{2} W^{T}(t) D^{T} D W(t) \\
= & \hbar_{1}^{T}(t) \Psi_{1}^{*}(P) \hbar_{1}(t)
\end{aligned}
$$

where $\hbar_{1}(t)=\left[\begin{array}{ll}\bar{X}^{T}(t) & W^{T}(t)\end{array}\right]^{T}, \quad \Phi=P\left(\bar{A}+B_{h} F\right)+$ $\left(\bar{A}+B_{h} F\right)^{T} P+h^{*} \varepsilon_{1} \bar{A}^{T} \bar{A}+h^{*} \varepsilon_{2} F^{T} B_{h}^{T} B_{h} F+h^{*}\left(\varepsilon_{1}^{-1}+\varepsilon_{2}^{-1}+\right.$ $\left.\varepsilon_{3}^{-1}\right) P B_{h} F F^{T} B_{h}^{T} P+L^{T} L$, and

$$
\Psi_{1}^{*}(P)=\left[\begin{array}{cc}
\Phi & P D \\
D^{T} P & \left(h^{*} \varepsilon_{3}-\gamma^{2}\right) D^{T} D
\end{array}\right]
$$

We know that the system is robustly stable with a disturbance attenuation $\gamma$ if $H(\bar{X}, W, t)<0$, which is equivalent to $\Psi_{1}^{*}(P)<0$, and then equivalent to the following algebraic Riccati inequality(ARI), if $h^{*} \varepsilon_{3}-\gamma^{2}<0$,

$$
\begin{aligned}
& P\left(\bar{A}+B_{h} F\right)+\left(\bar{A}+B_{h} F\right)^{T} P+h^{*} \varepsilon_{1} \bar{A}^{T} \bar{A} \\
& +h^{*} \varepsilon_{2} F^{T} B_{h}^{T} B_{h} F+h^{*}\left(\varepsilon_{1}^{-1}+\varepsilon_{2}^{-1}+\varepsilon_{3}^{-1}\right) P B_{h} F F^{T} B_{h}^{T} P \\
& +L^{T} L+\frac{1}{\gamma^{2}-h^{*} \varepsilon_{3}} P D D^{T} P<0
\end{aligned}
$$

Note that $D^{T} D=I$, we premultiply and postmultiply (48) by $P$ and get the following equivalent equation:

$$
\begin{aligned}
& \left(\bar{A}+B_{h} F\right) Y+Y\left(\bar{A}+B_{h} F\right)^{T}+h^{*} \varepsilon_{1} Y \bar{A}^{T} \bar{A} Y \\
& +h^{*} \varepsilon_{2} Y F^{T} B_{h}^{T} B_{h} F Y+h^{*}\left(\varepsilon_{1}^{-1}+\varepsilon_{2}^{-1}+\varepsilon_{3}^{-1}\right) B_{h} F F^{T} B_{h}^{T} \\
& +Y L^{T} L Y+\frac{1}{\gamma^{2}-h^{*} \varepsilon_{3}} D D^{T}<0
\end{aligned}
$$

According to the Schur Complement, the ARI in (49) is equivalent to the following LMI.

$$
\left[\begin{array}{cccc}
\Phi & Y \bar{A}^{T} & Y F^{T} B_{h}^{T} & Y L^{T} \\
\bar{A} Y & -\frac{1}{h^{*} \varepsilon_{1}} I & 0 & 0 \\
B_{h} F Y & 0 & -\frac{1}{h^{*} \varepsilon_{2}} I & 0 \\
L Y & 0 & 0 & -I
\end{array}\right]<0
$$

where $\Phi=\left(\bar{A}+B_{h} F\right) Y+Y\left(\bar{A}+B_{h} F\right)^{T}+h^{*}\left(\varepsilon_{1}^{-1}+\varepsilon_{2}^{-1}+\right.$ $\left.\varepsilon_{3}^{-1}\right) B_{h} F F^{T} B_{h}^{T}+\frac{1}{\gamma^{2}-h^{*} \varepsilon_{3}} D D^{T}<0$.

Given this theorem, we should notice that the matrix inequality is not linear and can not be easily converted to a LMI due to the term that contains $F F^{T}$ in $\Phi$. This difficulty 
is caused by the time delay $h^{*}$. Such that we cann't solve the matrix inequality (50) for $F$ and $Y$ simultaneously. We show how to determine the $F$ by two steps in the next section.

\section{E. Robust Formation Controller Design}

We design a state feedback $\mu(t)=K_{2} X_{e}(t-h)+K_{1} Z_{e}(t-$ $h)$, such that the system described by (41) is stabilized. First, we design $K_{1}$ such that $\mu^{\prime}(t)=K_{1} Z_{e}(t-h)$ stabilize the following velocity subsystem:

$$
\dot{Z}_{e}(t)=A_{\lambda} Z_{e}(t)+W(t)+\mu^{\prime}(t) .
$$

Remark 1: Given $\left\|A_{\Delta}\right\|_{\infty} \leq \sigma<\infty$, according to the Small Gain Theorem [21], system (51) with perturbation $W=A_{\Delta} Z_{e}$ is well-posed and internally stable for all $A_{\Delta} \in R H_{\infty}$ with $\left\|A_{\Delta}\right\|_{\infty} \leq 1 / \gamma$ if and only if $\left\|T_{Z_{e} W}\right\|_{\infty}<\gamma$, where $\gamma>0$, and $T_{Z_{e} W}$ is the closed-loop transfer matrix from $W$ to $Z_{e}$.

Theorem 2: Suppose the time delay $h$ satisfies $0 \leq h \leq h^{*}$ and $\dot{h}=0$. Let $P_{1}=P_{1}^{T}>0$ and $P_{2}<0$ be solutions of the following LMI:

$$
\Psi_{2}^{*}=\left[\begin{array}{ccc}
A_{\lambda}^{T} P_{1}+P_{1} A_{\lambda}+Q+I & P_{2} & P_{1} \\
P_{2}^{T} & -Q & 0 \\
P_{1} & 0 & -\gamma^{2} I
\end{array}\right]<0 .
$$

The control law $\mu^{\prime}(t)=K_{1} Z_{e}(t-h)$, where

$$
K_{1}=P_{1}^{-1} P_{2}<0 \text {, }
$$

robustly stabilizes the formation velocity subsystem (51), i.e., $\left\|T_{Z_{e} W}\right\|_{\infty}<\gamma$, where $\gamma \leq 1 /\left\|A_{\Delta}\right\|$.

Proof 2: Let the output of system (51) to be $Z_{e}$. We define a Lyapunov-Krasovskii functional as

$$
V_{2}\left[Z_{e}(t)\right]=Z_{e}^{T}(t) P_{1} Z_{e}(t)+\int_{t-h}^{t} Z_{e}^{T}(s) Q Z_{e}(s) d s,
$$

where $P_{1}=P_{1}^{T}>0, Q=Q^{T}>0$, and a Hamiltonian function as

$$
H\left(Z_{e}, W, t\right)=\dot{V}_{2}\left[Z_{e}(t)\right]+Z_{e}^{T}(t) Z_{e}(t)-\gamma^{2} W^{T}(t) W(t),
$$

then we can get derivative of the Lyapunov-Krasovskii functional $\dot{V}_{2}\left[Z_{e}(t)\right]$ as

$$
\begin{aligned}
& \dot{V}_{2}\left[Z_{e}(t)\right]=Z_{e}^{T}(t)\left[A_{\lambda}^{T} P_{1}+P_{1} A_{\lambda}+Q\right] Z_{e}(t) \\
& +Z_{e}^{T}(t-h) K_{1}^{T} P_{1} Z_{e}(t)+Z_{e}^{T}(t) P_{1} K_{1} Z_{e}(t-h) \\
& -Z_{e}^{T}(t-h) Q Z_{e}(t-h)+W^{T}(t) P_{1} Z_{e}(t)+Z_{e}^{T}(t) P_{1} W(t) .
\end{aligned}
$$

Therefore, by plugging $\dot{V}_{2}\left[Z_{e}(t)\right]$ into the Hamiltonian function in (55), we get

$$
\begin{aligned}
& H\left(Z_{e}, W, t\right)=Z_{e}^{T}(t)\left[A_{\lambda}^{T} P_{1}+P_{1} A_{\lambda}+Q\right] Z_{e}(t) \\
& +Z_{e}^{T}(t-h) K_{1}^{T} P_{1} Z_{e}(t)+Z_{e}^{T}(t) P_{1} K_{1} Z_{e}(t-h) \\
& -Z_{e}^{T}(t-h) Q Z_{e}(t-h)+W^{T}(t) P_{1} Z_{e}(t) \\
& +Z_{e}^{T}(t) P_{1} W(t)+Z_{e}^{T}(t) Z_{e}(t)-\gamma^{2} W^{T}(t) W(t) \\
& =\hbar_{2}^{T}(t) \Psi_{1}^{*} \hbar_{2}(t),
\end{aligned}
$$

where $\hbar_{2}(t) \doteq\left[\begin{array}{lll}Z_{e}^{T}(t) & Z_{e}^{T}(t-h) & W^{T}(t)\end{array}\right]^{T}$ and

$$
\Psi_{2}^{*} \doteq\left[\begin{array}{ccc}
A_{\lambda}^{T} P_{1}+P_{1} A_{\lambda}+Q+I & P_{1} K_{1} & P_{1} \\
K_{1}^{T} P_{1} & -Q & 0 \\
P_{1} & 0 & -\gamma^{2} I
\end{array}\right] .
$$

Let $P_{1}=P_{1}^{T}>0, P_{2}=P_{1} K_{1}<0$ (i.e. $K_{1}=P_{1}^{-1} P_{2}$ ) and $Q=$ $Q^{T}>0$ are feasible solutions for $\Psi_{2}^{*}<0$, then $H\left(Z_{e}, W, t\right)<$ 0 . Hamiltonian principle suggests that $H\left(Z_{e}, W, t\right)<0$ and $\left\|T_{Z_{e} W}\right\|_{\infty}<\gamma$, where $\gamma \leq 1 /\left\|A_{\Delta}\right\|$, are equivalent.

Now the velocity subsystem with perturbations is robustly stable under control $\mu^{\prime}(t)=K_{1} Z_{e}(t-h)$ for arbitrary bounded delay. Fixing $K_{1}$, we design $K_{2}$, such that $\mu(t)=K_{1} Z_{e}(t-$ $h)+K_{2} X_{e}(t-h)$ stabilizes the perturbed time-delay formation system (41). According to the theorem 1 , we choose $K_{2}$ as a negative definite symmetric matrix and then check whether we can find $Y, \varepsilon_{1}, \varepsilon_{2}$, and $\varepsilon_{3}$ from (50). Since $F$ is known, the inequality (50) becomes linear and it can be easily solved by Matlab LMI Toolbox.

\section{SIMULATION}

We carry out simulations for a group of six AUVs, use the AUV parameters provided in [18], and set the time step for simulation as $0.01 \mathrm{~s}$.

$m=200 \mathrm{~kg}, \mathscr{X}_{u}=-70, \mathscr{Y}_{v}=-100, \mathscr{Z}_{w}=-50, \mathscr{X}_{\dot{u}}=-62.5$, $\mathscr{Y}_{\dot{v}}=-70, \mathscr{Z}_{\dot{w}}=-50, \mathscr{K}_{p}=-0.8, \mathscr{M}_{q}=-0.9, \mathscr{N}_{r}=-0.4$,

$\mathscr{K}_{\dot{p}}=-200, \mathscr{M}_{\dot{q}}=-350, \mathscr{N}_{\dot{r}}=-500, I_{x}=203 \mathrm{Nms}^{2}$,

$I_{y}=587 \mathrm{Nms}^{2}, I_{z}=687 \mathrm{Nms}^{2}$.

The simulation is to show six AUVs tracking a sinusoidal line and keeping a polygon shape. Initial AUV positions and linear velocities are randomly generated in the following interval: $x \in(-10 \mathrm{~m}, 10 \mathrm{~m}), y \in(-10 \mathrm{~m}, 10 \mathrm{~m}), z \in$ $(30 \mathrm{~m}, 50 \mathrm{~m})$ and $u, v, w \in(-5 \mathrm{~m} / \mathrm{s}, 5 \mathrm{~m} / \mathrm{s})$. Euler angles and angular speeds initialized at zeros. The desired formation center trajectory is $(5 t, 0,40+5 \sin t)$. Jacobi vectors are defined as $\rho_{1}=\frac{1}{\sqrt{2}}\left(p_{2}-p_{1}\right), \rho_{2}=\frac{1}{\sqrt{2}}\left(p_{3}-p_{4}\right), \rho_{3}=\frac{1}{\sqrt{2}}\left(p_{5}-\right.$ $\left.p_{6}\right), \rho_{4}=\frac{1}{2}\left(p_{4}+p_{3}-p_{1}-p_{2}\right)$, and $\rho_{5}=\frac{1}{4}\left(p_{1}+p_{2}+p_{3}+\right.$ $\left.p_{4}-2 p_{5}-2 p_{6}\right)$. The desired values are $\rho_{1} \rightarrow[0,15,0]^{T}, \rho_{2} \rightarrow$ $[0,-15,0]^{T}, \rho_{3} \rightarrow[0,0,20]^{T} \quad \rho_{4} \rightarrow[0,0,-15]^{T}$, and $\rho_{5} \rightarrow$ $[0,0,0]^{T}$. The time delay is assumed to be $h=0.5 \mathrm{~s}$. Figure 1 shows that the six AUVs move along the desired trajectory and converge to the desired polygon shape. Figure 2,3, and 4 illustrate that linear velocities $u, v, w$ of the six AUVs all converge to their desired values seperately.

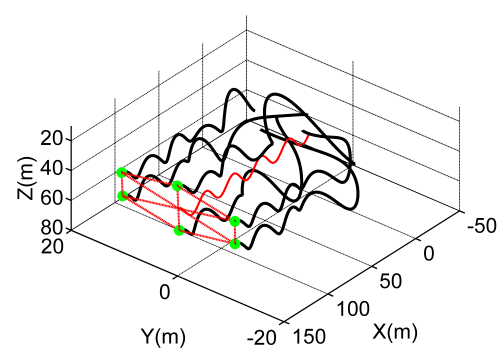

Fig. 1. Trajectory and formation of six AUVs $(h=0.5 \mathrm{~s})$.

\section{CONCLUSIONS}

This paper presented a robust formation controller design method for an AUV group with time delays. We studied 


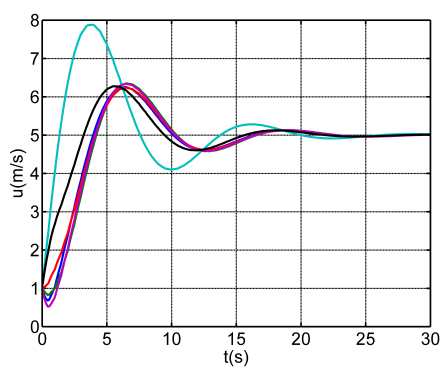

Fig. 2. Surge velocities of six AUVs $(h=0.5 \mathrm{~s})$.

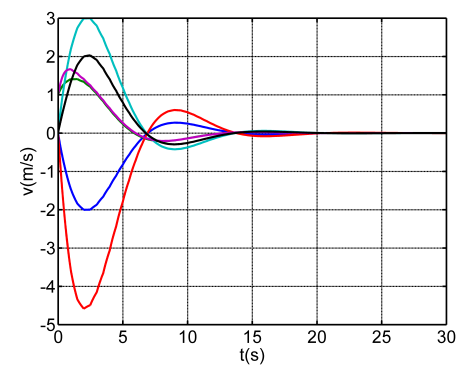

Fig. 3. Sway velocities of six AUVs $(h=0.5 \mathrm{~s})$.

the 6DOF motion model for a single AUV, split the orientation system from the collected dynamics, and designed the orientation controller based on feedback linearization. Using Jacobi transform, we derived the formation dynamics of AUVs, and decoupled the dynamics into a formation shape and a formation center. The robust formation controller tolerates perturbations and time delays, and guarantees that the AUVs achieve trajectory tracking and formation keeping simultaneously. Future work will include collision avoidance, obstacle avoidance, and extension of this centralized approach to a distributed approach.

\section{REFERENCES}

[1] R. Smith and F. Hadaegh, "Distributed estimation, communication and control for deep space formations," IET Control Theory Applications, vol. 1, no. 2, pp. 445 -451, march 2007.

[2] S. Emrani, A. Dirafzoon, and H. Talebi, "Adaptive distributed formation control of multiple autonomous underwater vehicles," in Control Applications (CCA), 2011 IEEE International Conference on, sept. 2011, pp. $693-698$.

[3] E. Yang and D. Gu, "Nonlinear formation-keeping and mooring control of multiple autonomous underwater vehicles," Mechatronics, IEEE/ASME Transactions on, vol. 12, no. 2, pp. 164 -178, april 2007.
[4] S. Fan, Z. Feng, and L. Lian, "Collision free formation control for multiple autonomous underwater vehicles," in OCEANS 2010 IEEE Sydney, may 2010, pp. $1-4$.

[5] W. Dong and J. A. Farrell, "Decentralized cooperative control of multiple nonholonomic dynamic systems with uncertainty," Automatica, vol. 45, pp. 706 - 710, 2009.

[6] Y. Wang, W. Yan, and J. Li, "Passivity-based formation control of autonomous underwater vehicles," IET Control Theory and Applications, vol. 6, no. 4, pp. 518-525, 2012.

[7] P. L. Kempker, A. C. Ran, and J. H. van Schuppen, "A formation flying algorithm for autonomous underwater vehicles," in 2011 50th IEEE Conference on Decision and Control and European Control Conference (CDC-ECC), Orlando, FL, USA, 2011, pp. 1293-1298.

[8] S. Kalantar and U. R. Zimmer, "Distributed shape control of homogeneous swarms of autonomous underwater vehicles," Autonomous Robots, vol. 22, no. 1, pp. 37-53, 2007.

[9] S. P. Hou and C. C. Cheah, "Can a simple control scheme work for a formation control of multiple autonomous underwater vehicles?" IEEE Transactions on Control Systems Technology, vol. 19, no. 5, pp. 10901101, 2011.

[10] A. P. Aguiar and J. P. Hespanha, "Trajectory-tracking and pathfollowing of underactuated autonomous vehicles with parametric modeling uncertainty," IEEE Transactions on Automatic Control, vol. 52, no. 8, pp. 1362-1379, 2007.

[11] E. B $\phi$ haug and K. Y. Pettersen, "Formation control of 6-dof eulerlagrange systems with restricted inter-vehicle communication," in Proceedings of the 45th IEEE Conference on Decision and Control, San Diego, CA, USA, 2006, pp. 5718-5723.

[12] D. J. Klein, P. Lee, K. A. Morgansen, and T. Javidi, "Integration of communication and control using discrete time kuramoto models for multivehicle coordination over broadcast networks," IEEE Journal on Selected Areas in Communications, vol. 26, no. 4, pp. 695 - 705, 2008.

[13] R. Ghabcheloo, A. P. Aguiar, A. Pascoal, C. Silvestre, I. Kaminer, and J. Hesphanha, "Coordinated path-following control of multiple underactuated autonomous vehicles in the presence of communication failures," in Proceedings of the 45th IEEE Conference on Decision and Control, San Diego, CA, USA, 2006, pp. 4345-4350.

[14] J. Ghommam and F. Mnif, "Coordinated path-following control for a group of underactuated surface vessels," IEEE Transactions on Industrial Electronics, vol. 56, no. 10, pp. 3951-3963, 2009.

[15] W. Dong and J. A. Farrell, "Formation control of multiple underactuated surface vessels," IET Control Theory and Applications, vol. 2, no. 12, pp. 1077-1085, 2008.

[16] H. Yang and F. Zhang, "Robust control of formation dynamics for autonomous underwater vehicles in horizontal plane," Journal of Dynamic Systems, Measurement, and Control, vol. 134, no. 3, pp. 0310091-0310097, 2012.

[17] T. I. Fossen, Gaudiance and Control of Ocean Vehicles, 1st ed. Chichester: John Wiley and Sons, 1994.

[18] G. Antonelli, Underwater Robots-Motion and Force Control of Vehicle-Manipulator Systems, 2nd ed. Berlin: Springer-Verlag, 2006.

[19] F. Zhang, "Geometric cooperative control of particle formations," IEEE Transaction on Automatic Control, vol. 55, no. 3, pp. 800-803, 2010.

[20] M. S. Mahmoud, Robust Control and Filtering for Time-Delay Systems. New York: Marcel-Dekker, 2000.

[21] K. Zhou and J. C. Doyle, Essentials of Robust Control, 1st ed. NJ,USA: Prentice Hall, 1997.

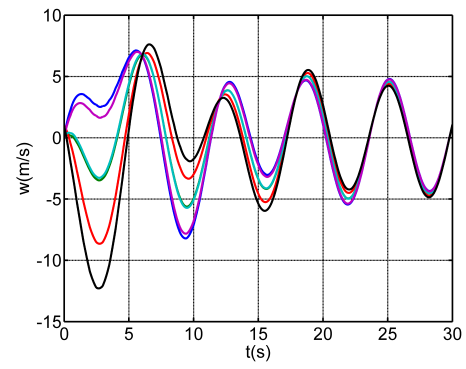

Fig. 4. Heaven velocities of six AUVs $(h=0.5 \mathrm{~s})$. 